Published in Europhysics Letters, 112, 67001 (2015)

\title{
Fermi liquid behaviour in weakly disordered metals close to a quantum critical point
}

\author{
George Kastrinakis \\ Institute of Electronic Structure and Laser (IESL), \\ Foundation for Research and Technology - Hellas (FORTH), P.O. Box 1527, Iraklio, Crete 71110, Greece*
}

(Dated: 6 November 2015)

\begin{abstract}
We calculate analytically the low temperature quasi-particle scattering rate, the conductivity, and the specific heat in weakly disordered metals close to a quantum critical point, via the use of a proper fluctuation potential $V(q, \omega)$ between the quasi-particles. We obtain typical Fermi liquid results proportional to $T^{2}$ and $T$ respectively, with prefactors which diverge as power laws of the control parameter $a$ upon approaching the critical point. The Kadowaki-Woods ratio is shown to be independent of $a$ (possibly times a logarithmic dependence on $a$ ) only for the case of threedimensional ferromagnetic fluctuations. Our results are consistent with experiments on the eight materials CeCoIn $5, \mathrm{Sr}_{3} \mathrm{Ru}_{2} \mathrm{O}_{7}, \mathrm{YbRh}_{2} \mathrm{Si}_{2}, \mathrm{La}_{2-x} \mathrm{Ce}_{x} \mathrm{CuO}_{4}, \mathrm{Tl}_{2} \mathrm{Ba}_{2} \mathrm{CuO}_{6+x}, \mathrm{CeAuSb}_{2}, \mathrm{YbAlB}_{4}$, and $\mathrm{CeRuSi}_{2}$.

PACS numbers: 72.10.-d,72.10.Di,72.15.Rn
\end{abstract}

Introduction. Quantum phase transitions take place at zero temperature and are due to the zero point quantum fluctuations. These fluctuations around the quantum critical point $(\mathrm{QCP})$ display scale invariance both in space and in time, and result in the influence of the QCP over a finite range of temperature $T$. Hence the effect of quantum criticality is detectable without actually reaching absolute zero $T$. Typically, some observables display diverging behaviour upon approaching the QCP.

In itinerant electron systems, the criticality parameter, which determines the proximity to the respective QCP, may depend on the electron filling factor, the pressure, or the magnetic field (which is related to filling, through the Zeeman term). A related review can be found in ref. [1]. Often these systems display non Fermi liquid behaviour, in the sense that e.g. the temperature dependence of various quantities measured differs from the standard Fermi liquid (FL) one [1].

Our work is motivated by a number of experiments on eight different materials [2 12], which display typical FL behaviour for appropriately low $T$. That is, quadratic in $T$ resistivity and linear in $T$ specific heat. However, the prefactors of these quantities appear to diverge as the respective QCP's are approached. We show, via analytic diagrammatic calculations, that these facts can be consistently understood as arising from the exchange of relevant fluctuations among the quasi-particles. Our approach assumes that we deal with weakly disordered metallic systems.

The model. We consider the Green's function

$$
G^{R, A}(k, \epsilon)=\frac{1}{\epsilon-\epsilon_{k}+\epsilon_{F} \pm i / 2 \tau_{o}},
$$

with $\epsilon_{k}$ the quasiparticle dispersion, $\epsilon_{F}$ the Fermi energy and $\tau_{o}$ the momentum relaxation time due to impurities. In the weak disorder regime [13, 14] $\epsilon_{F} \tau_{o} \gg 1 . \tau_{o}^{-1}$ is important as a regulator in our calculations. In fact, the characteristic FL $T^{2}$ dependence of $\operatorname{Im} \Sigma$ in eqs. (13), (14) is due to the finite $\tau_{o}^{-1}$.

The dominant electron-electron interaction is assumed to be the fluctuation potential (or fluctuation propagator) $15-20$

$$
V(q, \omega)=\frac{g}{-i \omega /\left(D q^{2}+r\right)+\xi^{2}\left(q-q_{0}\right)^{2}+a},
$$

with $g$ the coupling constant, $\xi$ the correlation length and $a$ measuring the distance from the QCP. The criticality parameter $a$ depends on e.g. the magnetic field $H$, as in the systems of interest mentioned below, like $a=h^{s}, h=\left|H / H_{c}-1\right|, s>0$, where $H_{c}$ is the critical field. The combination $\left(D q^{2}+r\right)$ in $V(q, \omega)$ is considered here for the first time. The factor $D q^{2}$ indicates disorder induced diffusion of the quasiparticles, with diffusion coefficient $D[14,21]$. This factor could also originate from antiferromagnetic damping, but this origin would not be consistent with our treatment of ferromagnetic fluctuations - c.f. below. The factor $r$ may originate from the inadvertent presence of elastic magnetic impurities in the samples [21], and should be equal to the relevant scattering rate $\tau_{S}^{-1}$. It can also originate from the fermiology of a clean system (without disorder). $q_{0}=0$ corresponds to ferromagnetic (FM) fluctuations, while finite $q_{0}$ to antiferromagnetic (AFM) fluctuations.

In general, $\xi$ and $a$ are expected to be related through an equation of the type [1, 16] $\xi^{-2}=d_{0} a+\beta T^{w}$, with $d_{0}, \beta, w$ constants. Below we will assume the Gaussian regime [15 20],

$$
\xi^{-2}=d_{0} a
$$

with $\beta=0$. However, for the purpose of our calculations, we will treat $\xi$ and $a$ as independent parameters, and consider the Gaussian regime relation in the final results. This procedure is entirely consistent, as can be seen from the details of the calculations below. 


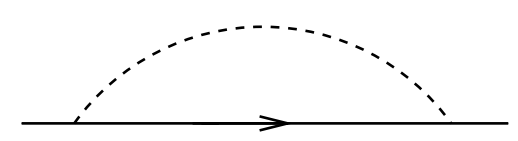

(a)

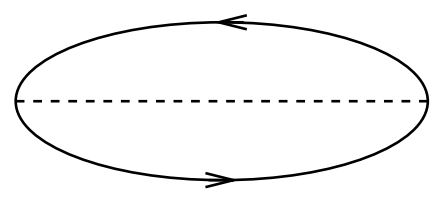

(b)

FIG. 1: Diagrams (a) for the self-energy and (b) for the free energy. The continuous lines are the fermion propagators, i.e. the Green's function of eq. (11), and the dashed line is the fluctuation mediated interaction $V(q, \omega)$.

Calculation of the scattering rate. We calculate the quasi-particle scattering rate as a function of $T \rightarrow 0$. For the self-energy $\Sigma$ we use the relation [13] (c.f. pg. 183)

$$
\begin{gathered}
\operatorname{Im} \Sigma^{R}(k, \epsilon)=\sum_{q} \int_{-\infty}^{\infty} d \omega \operatorname{Im} G^{R}(k-q, \epsilon-\omega) \\
\operatorname{Im} V^{R}(q, \omega)\{\operatorname{coth}(\omega / 2 T)+\tanh ((\epsilon-\omega) / 2 T)\},
\end{gathered}
$$

in order to calculate the scattering rate, which equals twice $\operatorname{Im} \Sigma$ - c.f. fig 1(a) for the corresponding Feynman diagram.

We present the calculation of $\operatorname{Im} \Sigma(k, \epsilon \rightarrow 0)$. In 3-D the integration over $q$ is $\sum_{q}=1 /(2 \pi)^{2} \int d q q^{2} \int_{-1}^{1} d x$, where $x=\cos \theta$, and $\theta$ is the angle between the vectors $\mathbf{k}$ and $\mathbf{q}$. From the factor $\operatorname{Im} G(k-q, \epsilon-\omega)$, we have the integral over $x$

$$
\begin{array}{r}
R(q, \omega)=\int_{-1}^{1} d x \operatorname{Im} G(k-q, \epsilon-\omega)=\int_{-1}^{1} \frac{d x s_{o}}{s_{o}^{2}+(c+b x)^{2}} \\
=\frac{1}{b}\left[\arctan \left(\frac{c+b}{s_{o}}\right)-\arctan \left(\frac{c-b}{s_{o}}\right)\right],
\end{array}
$$

where $s_{o}=1 / 2 \tau_{o}, c=\epsilon-\omega-\epsilon_{k}-q^{2} / 2 m+\epsilon_{F}$ and $b=$ $k q / m$. To be specific, we assume a parabolic dispersion relation $\epsilon_{k}=k^{2} / 2 m$. However, the precise form of the dispersion is not of particular importance. We have

$$
\operatorname{Im} V(q, \omega)=g \omega \frac{D q^{2}+r}{\omega^{2}+K_{q}^{2}}
$$

with $K_{q}=\left(D q^{2}+r\right)\left(\xi^{2}\left(q-q_{0}\right)^{2}+a\right)$. We consider the limit $\epsilon \rightarrow 0$. The function $A(x)=\operatorname{coth}(x)+\tanh (-x)$ satisfies $A(x \rightarrow 0)=1 / x+O(x)$ and $A(|x| \gg 1) \rightarrow 0$. Hence we evaluate the integral over $\omega$ as

$$
\int_{-2 T}^{2 T} d \omega\left(\frac{2 T}{\omega}\right) R(q, \omega) \frac{\omega}{\omega^{2}+K_{q}^{2}} \simeq \frac{8 T^{2}}{K_{q}^{2}} R(q),
$$

where $R(q)=R(q, \omega \simeq T)$, and $2 T<K_{q}$ is implied.

First we consider the case $q_{0}=0$. We see that the dominant contribution to the remaining integral $L$ over $q$ comes from finite $q>q_{1}=\sqrt{a} / \xi$

$$
L=\int_{q_{1}}^{q_{\max }} d q q^{2} \frac{\left(D q^{2}+r\right) R(q)}{q K_{q}^{2}} \simeq
$$

$\frac{R_{0}}{2 B}\left\{\frac{1}{a+\xi^{2} q^{2}}+\frac{D}{2 B} \ln \left(D q^{2}+r\right)-\frac{D}{2 B} \ln \left(a+\xi^{2} q^{2}\right)\right\}_{q_{1}}^{q_{\max }}$,

with $q_{\max }=1 / 2 \tau_{o} v_{F}, R_{0}=R(\bar{q})$ and $B=a D-r \xi^{2}$.

We consider in detail two different limiting cases, namely $r=0, D>0$ and $r>0, D=0$.

For the case $r=0, D>0$ we have

$L=\frac{R_{0}}{D}\left\{\frac{1}{a^{2}} \ln \left(\frac{q_{\max }}{q_{1}}\right)+\frac{1}{2 a}\left(\frac{1}{\xi^{2} q_{\max }^{2}+a}-\frac{1}{\xi^{2} q_{1}^{2}+a}\right)\right\}$.

This yields $L \propto 1 / a^{2}$. Then, for the case $r>0, D=0$ we have

$$
L=-\frac{R_{0}}{2 \xi^{2} r}\left\{\frac{1}{\xi^{2} q_{\max }^{2}+a}-\frac{1}{\xi^{2} q_{1}^{2}+a}\right\} .
$$

Here, $L \propto 1 /\left(\xi^{2} a\right)$.

To estimate $R_{0}$ we consider the relation $\arctan (x)-$ $\arctan (y)=\arctan ((x-y) /(1+x y))$, which yields $R(q) \simeq$ $\arctan \left(2 k q / m s_{o}\right)$. The most relevant momenta are $k \simeq$ $k_{F}$, thus yielding $k / m=v_{F}$. Then we take $\bar{q}=q_{\max } / 2$ and we obtain $R_{0} \simeq \arctan (1)=\pi / 4$.

Next, we turn to the case of finite $q_{0}$ and, as above, we consider $q>q_{1}=\sqrt{a} / \xi$ and the limit $a \rightarrow 0$.

We have for all $r, D$

$$
\begin{gathered}
L=\int_{q_{1}}^{q_{\max }} d q \frac{q R(q)}{\left(D q^{2}+r\right)\left(a+\xi^{2}\left(q-q_{0}\right)^{2}\right)^{2}} \\
\simeq \frac{q_{0} R_{0}}{2\left(D q_{0}^{2}+r\right) \xi a}\left\{\frac{1}{\sqrt{a}} \arctan \left(\frac{\xi\left(q-q_{0}\right)}{\sqrt{a}}\right)+\frac{\xi\left(q-q_{0}\right)}{a+\xi^{2}\left(q-q_{0}\right)^{2}}+O(\sqrt{a})\right\}_{q_{1}}^{q_{\max }}
\end{gathered}
$$


We now turn to 2-D. Again, $\theta$ is the angle between the vectors $\mathbf{k}$ and $\mathbf{q}$. From the factor $\operatorname{Im} G(k-q, \epsilon-\omega)$, we have the angular integral $I(q, \omega)=\int_{0}^{2 \pi} \frac{d \theta s_{o}}{s_{o}^{2}+(c+b \cos \theta)^{2}}$ with $s_{o}, c, b$ as above. For small $\omega$ and $k \simeq k_{F}, \epsilon \rightarrow 0$ we can make the approximation $I(q, \omega) \simeq \frac{2 \pi}{s_{o}}=I_{0}$. As above, the integral over $\omega$ is $\int_{-2 T}^{2 T} d \omega(2 T / \omega)\left(\omega I_{0}\right) /\left(\omega^{2}+\right.$ $\left.K_{q}^{2}\right) \simeq 8 T^{2} I_{0} / K_{q}^{2}$. Then, we have the remaining integral $L_{2}$ over $q$ in 2 -D

$$
L_{2}=\int_{q_{1}}^{q_{\max }} d q q \frac{I_{0}}{\left(D q^{2}+r\right)\left(a+\xi^{2}\left(q-q_{0}\right)^{2}\right)^{2}} .
$$

We notice that the integrand is very similar to the 3$\mathrm{D}$ case of eq. (11), and differing only in the factor $I_{0}$. Hence, we obtain the same scaling of $\operatorname{Im} \Sigma$ with $a$ as in 3-D.

In 3 dimensions the final result is

$$
\operatorname{Im} \Sigma\left(k_{F}, \epsilon=0, T, a\right)=g /\left(2 \pi v_{F}\right) f_{3}\left(a, q_{0}\right) T^{2},
$$

with $v_{F}$ the Fermi velocity. The function $f_{3}\left(a, q_{0}\right)$ is given in Table 1. Therein $l_{0}=q_{0} /\left(D q_{0}^{2}+r\right)$.

In 2 dimensions we have

$$
\operatorname{Im} \Sigma\left(k_{F}, \epsilon=0, T, a\right)=\left(8 g \tau_{o} / \pi\right) f_{2}\left(a, q_{0}\right) T^{2} .
$$

It turns out that $f_{2}\left(a, q_{0}\right)=f_{3}\left(a, q_{0}\right)$, so the prefactor dependence on $a$ is the same as in 3-D. We note that the above dependence on $a, \xi$ and $T$ is valid for all $k$ of the order of $q_{\max }$ or greater. For $k$ away from $k_{F}$ only the prefactors change.

A non-FL result $\operatorname{Im} \Sigma \propto \sqrt{T}$ was obtained in [20] for clean metallic systems close to a FM QCP. We emphasize that herein we treat weakly disordered systems instead c.f. the comment on $\tau_{o}^{-1}$ following eq. (1) above.

Calculation of the conductivity. We consider the total quasi-particle scattering rate

$$
\tau^{-1}(T, a)=\tau_{o, i}^{-1}+2 \operatorname{Im} \Sigma\left(k_{F}, \epsilon=0, T, a\right)=2 S,
$$

with $\tau_{o, i}^{-1}$ due to impurity scattering.

We calculate the total conductivity $\sigma$ from an infinite series of diagrams involving disorder and $V(q, \omega)$. The $n$-th term of the conductivity series, shown in fig. 2, comprises $n$ impurity scattering lines in parallel. We recall that two impurity lines crossing each other introduce a small factor $1 / \epsilon_{F} \tau_{o} \ll 1$ - c.f. refs. [13, 14]. Hence we ommit any such diagram. In ref. [22] we summed up to infinite order another diagrammatic conductivity series, which includes disorder and interactions (and yields experimentally observed positive giant magnetoresistance). The Green's function here is taken as

$$
G_{*}^{R, A}(k, \epsilon)=\frac{1}{\epsilon-\epsilon_{k}+\epsilon_{F} \pm i S},
$$

i.e. it includes the self-energy of eq. (15) due to the fluctuation potential $V(q, \omega)$. Due to momentum conservation, the momenta in the upper and lower lines of

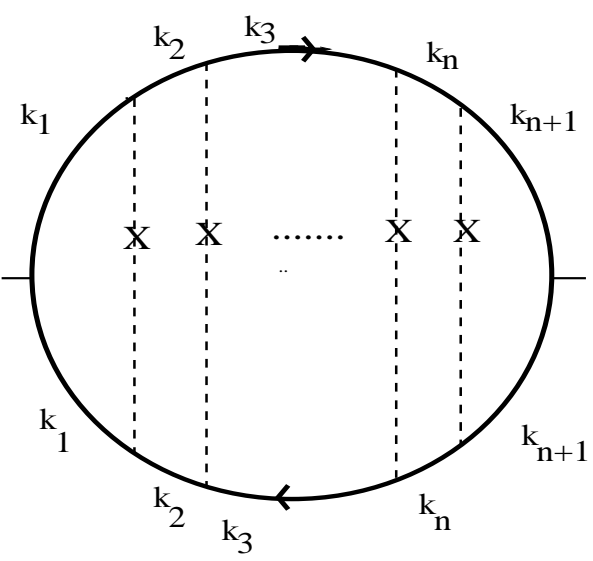

FIG. 2: Feynman diagram for the $n$-th term of the conductivity series. The dashed lines with crosses stand for impurity scattering. There are $n$ such impurity lines inside the conductivity bubble. The Green's function here is given by eq. (16).

the $m$-th pair of $G_{*}^{R} G_{*}^{A}$ are the same. The major contribution to these diagrams comes from assuming for the various vectors $k_{1}=k_{2}=k_{3}=\ldots=k_{n}=k_{n+1}$. Also $\epsilon=0$.

Ommitting vertex corrections, the $n$-th term of this series is given by $\left(\sigma=\sum_{n=0}^{\infty} \sigma_{n}\right)$

$$
\begin{aligned}
\sigma_{n}= & u_{o}^{n} \frac{2 e^{2}}{m^{2}} \int d \mathbf{k} k^{2}\left(G_{*}^{R}(k, 0) G_{*}^{A}(k, 0)\right)^{n+1} \\
& =u_{o}^{n} \frac{4 N_{F} e^{2}}{m} \int_{-\epsilon_{F}}^{\infty} d x \frac{\epsilon_{F}+x}{\left(x^{2}+S^{2}\right)^{n+1}}
\end{aligned}
$$

where $x=\epsilon_{k}-\epsilon_{F}, N_{F}$ is the density of states at the Fermi level, $e$ is the charge of the electron, $m$ is the mass of the electron, $u_{o}=n_{i m p} V_{i m p}^{2}$, with $n_{i m p}$ the concentration of impurities, $V_{i m p}$ the typical value of the impurity scattering potential. Summing up this series, and considering the energy scale $E_{0}>\epsilon_{F}$, the result is

$$
\begin{aligned}
\sigma=\frac{4 e^{2} N_{F}}{m}\left\{\frac{\epsilon_{F}}{\sqrt{S^{2}-u_{o}}}\right. & {\left[\frac{\pi}{2}+\arctan \left(\frac{\epsilon_{F}}{\sqrt{S^{2}-u_{o}}}\right)\right] } \\
+ & \left.\frac{1}{2} \ln \left(\frac{E_{0}^{2}+S^{2}}{\epsilon_{F}^{2}+S^{2}-u_{o}}\right)\right\} .
\end{aligned}
$$

For $E_{0} \sim O\left(\epsilon_{F}\right)$ and $\epsilon_{F}>S$ we may approximate $\sigma=$ $4 \pi e^{2} N_{F} \epsilon_{F} /\left(m \sqrt{S^{2}-u_{o}}\right)$.

As the resistivity $\rho=1 / \sigma$ comes out proportional to the scattering rate, these results compare very favorably with data on $\mathrm{CeCoIn}_{5}$,2, 3], $\mathrm{Sr}_{3} \mathrm{Ru}_{2} \mathrm{O}_{7}$ [4] and $\mathrm{YbRh}_{2} \mathrm{Si}_{2}$ [5, 6], where $\rho$ displays typical FL $T^{2}$ dependence

$$
\rho(T, a)=\rho_{0}+A(a) T^{2},
$$

with $\rho_{0}=$ const., and $A(a)$ diverging around the QCP, i.e. around $a=0$, as in our result. With all the results of Table 1 in mind, we may concretely assume that the 
Gaussian 3-D case $q_{0}=0, r=0, D>0$ applies (with the alternative being the $3-\mathrm{D}$ case $q_{0}=0, r>0, D=0$, and $\xi$ not scaling with $a$, as mentioned below). Assuming the scaling $A(a) \propto a^{-2}$, as in the case $q_{0}=0(\mathrm{FM})$ above, the diverging $A(a \rightarrow 0)$ in $\mathrm{CeCoIn}_{5}$ yields $2 s=1.29 \pm 0.1$ for the resistivity and $2 s=1.34 \pm 0.1$ for the thermal resistivity [2, 3]. Hence, for $\mathrm{CeCoIn}_{5}$, the WiedemannFranz law, i.e. a constant ratio of the thermal conductivity $\kappa$ over the electrical conductivity $\sigma$, times $T$, in the low $T$ limit, i.e. $\kappa / \sigma T=$ const., is obeyed [2]. These experimental results are easily understood in the frame of our calculation: conduction electrons carry both charge and heat, while interacting via $V(q, \omega)$ (only small energy transfer is involved with this $V(q, \omega))$.

The same scaling $A(a) \propto a^{-2}$ was also found to fit data in $\mathrm{La}_{2-x} \mathrm{Ce}_{x} \mathrm{CuO}_{4}$ [7] with $2 s=0.38$, in overdoped $\mathrm{Tl}_{2} \mathrm{Ba}_{2} \mathrm{CuO}_{6+x}$ [8] with $2 s=0.62$, in $\mathrm{CeAuSb}_{2}$ [9] with $2 s=1.0$, and in $\mathrm{YbAlB}_{4}[10]$ with $2 s=0.50$.

In principle, it should be possible to probe the scattering rate through angle-resolved photoemission (ARPES) experiments (which require, of course, that the materials in question be adequately cleavable).

Calculation of the specific heat. We calculate the specific heat $C(T)=-T \partial^{2} F(T) / \partial T^{2}=\gamma T$, through the free energy $F(T)$ - c.f. fig $1(\mathrm{~b})$ for the relevant Feynman diagram. To lowest order in $V(q, \omega)$ we have

$$
\begin{array}{r}
F(T)=T^{2} \sum_{k, q, \epsilon_{l}, \omega_{m}} V\left(q, i \omega_{m}\right) G\left(k+q, i \epsilon_{l}+i \omega_{m}\right) G\left(k, i \epsilon_{l}\right) \\
=T \sum_{q, \omega_{m}} V\left(q, i \omega_{m}\right) \Pi\left(q, i \omega_{m}\right)
\end{array}
$$$$
F(T)=-\Gamma(T) T^{2}
$$

First, we consider the case $q_{0}=0$. In 3 -D we have, with $c_{3}=\frac{g N_{F}}{2 \pi^{3}}$ and $q_{a}=\min \left\{\sqrt{T /(a D)},\left(T /\left(D \xi^{2}\right)\right)^{1 / 4}\right\}$, the (20)remaining integral over $q$

$$
\Gamma(T)=c_{3} \int_{q_{a}}^{q_{\max }} \frac{d q q^{2}}{\left(D q^{2}+r\right) Y_{q}^{2}}=\frac{c_{3}}{2 B^{2}}\left\{\frac{q B}{a+\xi^{2} q^{2}}-2 \sqrt{r D} \arctan \left(\frac{q \sqrt{D}}{\sqrt{r}}\right)+\frac{a D+r \xi^{2}}{\xi \sqrt{a}} \arctan \left(\frac{\xi q}{\sqrt{a}}\right)\right\}_{q_{a}}^{q_{\max }}
$$

To proceed, we take the limit $T \rightarrow 0$ first and then the limit $a \rightarrow 0$, in order the extract the coefficient $\gamma$.

Likewise, in 2-D, with $c_{2}=g N_{F} / \pi^{2}$ we have for $q_{0}=0$

$$
\begin{array}{r}
\Gamma(T)=c_{2} \int_{q_{a}}^{q_{\max }} \frac{d q q}{\left(D q^{2}+r\right) Y_{q}^{2}} \\
=\frac{c_{2}}{2 B}\left\{\frac{1}{a+\xi^{2} q^{2}}+\frac{1}{B} \ln \left(\frac{D q^{2}+r}{a+\xi^{2} q^{2}}\right)\right\}_{q_{a}}^{q_{\max }} .
\end{array}
$$

Here the Matsubara energies are $\epsilon_{l}=(2 l+1) \pi T$ and $\omega_{m}=2 m \pi T, D_{o}=v_{F}^{2} \tau_{o} / d(d=2,3$ according to dimensionality) and [14] $\Pi\left(q, i \omega_{m}\right)=T \sum_{k, \epsilon_{l}} G\left(k+q, i \epsilon_{l}+\right.$ $\left.i \omega_{m}\right) G\left(k, i \epsilon_{l}\right)=N_{F}\left(1-\tau_{o}\left(D_{o} q^{2}+\left|\omega_{m}\right|\right)\right)$.

The integrand $P(q, \omega)=V(q, \omega) \Pi(q, \omega)$ has a branch cut for $\operatorname{Im} \omega=0$, which comes from $\operatorname{Im} V(q, \omega)$ (in which $\left.-i \omega \rightarrow+\left|\omega_{m}\right|\right)$. We ommit the $q, \omega$ dependence of $\Pi(q, \omega)$. Then, via Cauchy's residue theorem, we obtain $W=T \sum_{\omega_{m}} P\left(q, i \omega_{m}\right)=-\int_{-\infty}^{\infty} \frac{d \omega}{\pi} n_{B}(\omega) \operatorname{Im} P(q, \omega)$. The Bose distribution function $n_{B}(\omega)=1 /\left(e^{(\omega / T)}-1\right)$ is commonly approximated [21] as $n_{B}(\omega)=(T / \omega) \theta(T-$ $|\omega|)$. We first carry out the $\omega$ integration and then the ntegration over the momentum $q$ in 2 and $3 \mathrm{D}$.

Setting $Y_{q}=\xi^{2}\left(q-q_{0}\right)^{2}+a$ we obtain

$$
\begin{array}{r}
W \simeq-\int_{-T}^{T} d \omega\left(\frac{T}{\omega}\right) \frac{\omega}{\left(D q^{2}+r\right)} \frac{1}{Y_{q}^{2}+\omega^{2} /\left(D q^{2}+r\right)^{2}} \\
=-\frac{2 T}{Y_{q}} \arctan \left(\frac{T}{\left(D q^{2}+r\right) Y_{q}}\right)
\end{array}
$$

We consider the low $T$ regime $T<\left(D q^{2}+r\right) Y_{q}$ and, as a result, $F(T)$ satisfies

Next we turn to the case of finite $q_{0}$. We set $X_{0}=$ $D q_{0}^{2}+r$. Further, the minimum $q=q_{\text {min }}$ should now satisfy $q_{\min }=\sqrt{T / D} /\left(\xi \max \left\{q_{0}, q_{\max }\right\}\right)$. In 3-D

$$
\Gamma(T)=c_{3} \int_{q_{\min }}^{q_{\max }} \frac{d q q^{2}}{\left(D q^{2}+r\right) Y_{q}^{2}}=\frac{c_{3} q_{0}^{2}}{2 \xi X_{0} a}\left\{\frac{1}{\sqrt{a}} \arctan \left(\frac{\xi\left(q-q_{0}\right)}{\sqrt{a}}\right)+\frac{\xi\left(q-q_{0}\right)}{a+\xi^{2}\left(q-q_{0}\right)^{2}}+O(\sqrt{a})\right\}_{q_{\min }}^{q_{\max }}
$$


and 2 respectively. Due to phase space considerations, for $q_{0}=0$ we have $\gamma_{2 D} \propto \gamma_{3 D} / \sqrt{a}$.

These results are consistent with $\mathrm{CeCoIn}_{5}$ data [3] as a function of $a(H)$. They are also consistent with $\mathrm{YbRh}_{2} \mathrm{Si}_{2}$ [5] and Ge-doped $\mathrm{YbRh}_{2} \mathrm{Si}_{2}$ data [6] (c.f. fig. 2 therein), with $\mathrm{Sr}_{3} \mathrm{Ru}_{2} \mathrm{O}_{7}$ data [1], and with $\mathrm{CeRuSi}_{2}$ data [12].

Kadowaki-Woods ratio. The scaling of the Kadowaki-Woods (KW) ratio $A / \gamma^{2}$ in $3-\mathrm{D}$ and $2-\mathrm{D}$ is shown in Tables 1 and 2 respectively. Upon assuming the Gaussian regime, c.f. eq. (3), the $\mathrm{KW}$ ratio is constant only for the 3-D case $q_{0}=0, r=0, D>0$, modulo the logarithmic in $a$ divergence. However, if, alternatively, $\xi$ and $a$ were independent parameters, and $\xi$ would not scale with $a$, the KW ratio would be constant only for the 3 -D case $q_{0}=0, r>0, D=0$. In all other cases either the ratio goes to zero for small $a$, or there are no diverging prefactors $A, \gamma$, contrary to the experiments.

Also we mention that substituting the factor $\left(D q^{2}+r\right)$ in eq. (2) by $\left(v_{o} q\right)$, i.e. the usual ferromagnetic damping with $v_{o}$ a constant, does not yield a (quasi-)constant $\mathrm{KW}$ ratio both in 3-D and 2-D.

An $a$-independent $\mathrm{KW}$ ratio was observed in $\mathrm{CeCoIn}_{5}$ 3], $\mathrm{YbRh}_{2} \mathrm{Si}_{2}$ [5], Ge-doped $\mathrm{YbRh}_{2} \mathrm{Si}_{2}$ [6], and $\mathrm{YbAlB}_{4}$ 10] though (in most cases) in a more restricted range of $H$ than the scaling of the coefficient $A(a)$. E.g. in [6] the KW ratio increases as the control parameter $a \rightarrow 0$, which is consistent with the logarithmic dependence on a.

For the other materials mentioned above, the experimental data are incompletely known with respect to the KW ratio. Therefore, the possibility exists that they fall in some other case, among the ones mentioned in Tables 1 and 2 , such that the KW ratio is not constant.

In ref. [17], using a different approach, diverging FL prefactors were obtained both for the resistivity and the specific heat. However, the results differ from ours. Therein, the KW ratio is constant only for 2-D FM fluctuations.

A number of experiments, probing quantities other than the above mentioned, suggest AFM behavior in $\mathrm{CeCoIn}_{5}$ [2, 23]. A possible explanation is that both FM and AFM fluctuations coexist in this material, with $A(a)$ and $\gamma(a)$ being determined dominantly by FM fluctuations.

We also consider an interaction with peaks at specific wavevectors $\vec{q}_{0 i}$ (AFM case) $V_{*}(q, \omega)=$ $\sum_{i=1}^{n_{d}} g /\left\{-i \omega /\left(D q^{2}+r\right)+\xi^{2}\left(\vec{q}-\vec{q}_{0 i}\right)^{2}+a\right\}$. In 2-D for tetragonal symmetry $n_{d}=4$ and in 3-D for cubic symmetry $n_{d}=6$. For small $a$, in 2-D and 3-D the potential $V_{*}(q, \omega)$ gives the same scaling of the prefactors as for the finite $q_{0}$ cases above.

Overview. In all, a consistent Fermi liquid description emerges from these calculations. The renormaliza- tion of the fermions due to $V(q, \omega)$ leads back to the FL fixed point in a low- $T$ part of the phase diagram. This is consistent with experiments - e.g. c.f. fig. 3 of ref. [2] for the case $T^{2}$. The prefactors for the scattering rate, the resistivity and the specific heat diverge as power laws of the criticality parameter $a$. According to our calculations, the Kadowaki-Woods ratio is constant only for 3-D FM $\left(q_{0}=0\right)$ fluctuations (possibly times the logarithmic in $a$ divergence).

We did not calculate explicitly the effective mass $m_{*}$ of the electrons. Experiments in $\mathrm{Sr}_{3} \mathrm{Ru}_{2} \mathrm{O}_{7}$ 24] have indicated the absence of a magnetic field $H$ dependent renormalization of $m_{*}$ (definitely so for five out of the six bands). This result is not inconsistent with our calculations, where the diverging overall prefactors, as a function of $a$, come from the small $q$ dependence of the potential $V(q, \omega)$, and yield a uniform $a$ dependence within the Fermi surface (c.f. the comment after eq. (14)).

Finally, we comment on the linear in $T$ resistivity $\rho$ displayed by $\mathrm{Sr}_{3} \mathrm{Ru}_{2} \mathrm{O}_{7}$ [4] and $\mathrm{CeCoIn}_{5}[2]$ in the vicinity of the $\mathrm{QCP}$ and for not very low $T$. In ref. [25] we developed a fully microscopic FL model with a strong van Hove singularity (or, strong peak in the density of states), located at a characteristic energy $\epsilon_{v H}$ close to the Fermi level $\mu$. This yields a quasi-particle scattering rate which is linear in $T$ for $T>\left(\mu-\epsilon_{v H}\right) / 4$. The model works very well for the cuprates, and this is how the linear in $T$ resistivity of $\mathrm{La}_{2-x} \mathrm{Ce}_{x} \mathrm{CuO}_{4}$ [7] can be understood, given the small difference $\mu-\epsilon_{v H}$ for many cuprates [26], as shown by ARPES expts. Such expts. 27] on $\mathrm{Sr}_{3} \mathrm{Ru}_{2} \mathrm{O}_{7}$ indeed yielded $\mu-\epsilon_{v H}=4 \mathrm{meV}$, in agreement with our model [25]. It is fair to attribute the linear in $T$ resistivity of $\mathrm{CeCoIn}_{5}$ to the same mechanism, i.e. originating from a van Hove singularity, which resides close to the Fermi surface in the vicinity of the QCP. Moreover, it is reasonable to view the regime $\rho \propto T^{b}$, $1<b<2$, displayed by $\mathrm{Sr}_{3} \mathrm{Ru}_{2} \mathrm{O}_{7}$ [4] and $\mathrm{CeCoIn}_{5}$ [2], as a smooth transient between $b=1$ and $b=2$.

* e-mail : kast@iesl.forth.gr ; giwkast@gmail.com

[1] v. Löhneysen H., Rosch A., Vojta M. and Wölfle P., Rev. Mod. Phys. 79, (2007) 1015.

[2] Paglione J. et al., Phys. Rev. Lett. 97, (2006) 106606.

[3] Bianchi A. et al., Phys. Rev. Lett. 91, 257001 (2003).

[4] Grigera S.A. et al., Science 294, 329 (2001).

[5] Gegenwart P. et al., Phys. Rev. Lett. 89, 056402 (2002).

[6] Gegenwart P. et al., Phys. Rev. Lett. 94, 076402 (2005).

[7] Butch N.P. et al., PNAS 109, 8440 (2012).

[8] Shibauchi T. et al., PNAS 105, 7120 (2008).

[9] Balicas L. et al., Phys. Rev. B 72, 064422 (2005).

[10] Nakatsuji S. et al., Nature Phys. 4, 603 (2008).

[11] Rost A.W. et. al., Science 325, 160 (2009).

[12] Flouquet J. et al., Physica B 319, 251 (2002).

[13] Abrikosov A. A., Gorkov L. P. and Dzyaloshinski I. E., 
TABLE I: Table 1. Coefficients $f_{3}\left(a, q_{0}\right), \gamma$ and scaling of the Kadowaki-Woods ratio $A / \gamma^{2}\left(A \propto f_{3}\right)$ in 3 -D as a function of the criticality parameter $a \ll 1$ and of the correlation length $\xi$. Here $c_{3}=g N_{F} /\left(2 \pi^{3}\right), B=a D-r \xi^{2}, q_{m a x}=1 / 2 \tau_{o} v_{F}$, $q_{\min }=\sqrt{T / D} /\left(\xi \max \left\{q_{0}, q_{\max }\right\}\right), q_{1}=\sqrt{a} / \xi, l_{0}=q_{0} /\left(D q_{0}^{2}+r\right)$, and $X_{0}=D q_{0}^{2}+r$. Upon assuming the Gaussian regime, c.f. eq. (3), the Kadowaki-Woods ratio is constant for the case $q_{0}=0, r=0, D>0$, modulo the logarithmic in $a$ divergence. In this case, the argument of the logarithm is $\left(q_{\max } / a \sqrt{d_{o}}\right)$. C.f. text.

\begin{tabular}{|c|c|c|c|}
\hline $3-D$ & $f_{3}\left(a, q_{0}\right)$ & $\gamma$ & scaling of $A / \gamma^{2}$ \\
\hline$q_{0}=0, r=0, D>0$ & $\frac{1}{a^{2} D}\left[\ln \left(\frac{\xi q_{\max }}{\sqrt{a}}\right)-\frac{1}{4}\right.$ & $\frac{c_{3} \pi}{2 \xi D a^{3 / 2}}$ & $a \xi^{2} \ln \left(\xi q_{\max } / \sqrt{a}\right)$ \\
\hline$q_{0}=0, r>0, D=0$ & $\frac{1}{4 \xi^{2} r a}$ & $\frac{c_{3} \pi r \xi}{2 B^{2} \sqrt{a}}$ & constant $\xi^{4}$ \\
\hline$q_{0}>q_{\max }>q_{1}>0$ & $\frac{l_{0}}{a \xi^{2}}\left\{\frac{1}{q_{\max }-q_{0}}-\frac{1}{q_{1}-q_{0}}\right\}$ & $\frac{c_{3} q_{0}}{X_{0} \xi^{2} a}\left(\frac{1}{q_{\max }-q_{0}}-\frac{1}{q_{\min }-q_{0}}\right)$ & $a \xi^{2}$ \\
\hline$q_{\max }>q_{0}>q_{1}>0$ & $\frac{l_{0} \pi}{2 \xi a^{3 / 2}}$ & $\frac{c_{3} q_{0}^{2} \pi}{X_{0} \xi a^{3 / 2}}$ & $a^{3 / 2} \xi$ \\
\hline
\end{tabular}

TABLE II: Table 2. Coefficients $f_{2}\left(a, q_{0}\right), \gamma$ and scaling of the Kadowaki-Woods ratio $A / \gamma^{2}\left(A \propto f_{2}\right)$ in 2-D as a function of the criticality parameter $a \ll 1$ and of the correlation length $\xi$. Here $c_{2}=g N_{F} / \pi^{2}$. C.f. caption of Table 1 and text.

\begin{tabular}{|c|c|c|c|c|c|c|}
\hline $2-\mathrm{D}$ & \multicolumn{3}{|c|}{$f_{2}\left(a, q_{0}\right)$} & \multicolumn{2}{|r|}{$\gamma$} & scaling of $A / \gamma^{2}$ \\
\hline$q_{0}=0, r=0, D>0$ & $\frac{1}{a^{2} D}$ & $\ln \left(\frac{\xi q_{m}}{\sqrt{c}}\right.$ & $-\frac{1}{4}$ & \multicolumn{2}{|r|}{$\frac{c_{2}}{D a}\left\{\frac{1}{\xi^{2} q_{\max }^{2}}-\frac{1}{a}\right\}$} & $a^{2} \ln \left(\xi q_{\max } / \sqrt{a}\right)$ \\
\hline$q_{0}=0, r>0, D=0$ & \multicolumn{3}{|c|}{$\frac{1}{4 \xi^{2} r a}$} & $\frac{c_{2}}{2 r \xi^{2}}$ & $\left.\frac{1}{a}-\frac{1}{r \xi} \ln \left(\frac{a}{\xi^{2} q_{\max }^{2}}\right)\right\}$ & $a \xi^{2}$ \\
\hline$q_{0}>q_{\max }>q_{1}>0$ & $\frac{l_{0}}{a \xi^{2}}$ & $\frac{1}{q_{\max }-q_{0}}$ & $\frac{1}{q_{1}-q_{0}}$ & $\frac{c_{2}}{\xi^{2} a}$ & $\frac{1}{q_{\max }-q_{0}}-\frac{1}{q_{\min }-q_{0}}$ & $a \xi^{2}$ \\
\hline$q_{\max }>q_{0}>q_{1}>0$ & \multicolumn{3}{|c|}{$\frac{l_{0} \pi}{2 \xi a^{3 / 2}}$} & \multicolumn{2}{|r|}{$\frac{c_{2} \pi}{\xi a^{3 / 2}}$} & $a^{3 / 2} \xi$ \\
\hline
\end{tabular}

Methods of Quantum Field Theory in Statistical Physics, Prentice-Hall (Cliffwoods, NY), (1964).

[14] Lee P.A. and Ramakrishnan T.V., Rev. Mod. Phys. 57, (1985) 287.

[15] Hertz J., Phys. Rev. B 14, 1165 (1976).

[16] Millis A.J., Phys. Rev. B 48, 7183 (1993).

[17] Millis A.J., Schofield A.J., Lonzarich G.G. and Grigera S.A., Phys. Rev. Lett. 88, 217204 (2002).

[18] Kim Y.B. Millis A.J., Phys. Rev. B 67, 085102 (2003).

[19] Chubukov A.V., Galitski V.M. Yakovenko V.M., Phys. Rev. Lett. 94, 046404 (2005).

[20] Maslov D.L. Chubukov A.V., Phys. Rev. B 79, 075112 (2009).

[21] Kastrinakis G., Phys. Rev. B. 72, 075137 (2005); in this work it was shown that the $T \rightarrow 0$ saturation of the electron dephasing rate, observed in numerous expts., can be attributed to the elastic scattering from magnetic impurities.

[22] Kastrinakis G., Europhys. Lett. 42, 345 (1998).

[23] Koutroulakis G. et al., Phys. Rev. Lett. 104, 087001 (2010).

[24] Mercure J.-F. et al., Phys. Rev. B 81, 235103 (2010).

[25] Kastrinakis G., Physica C, 340, 119 (2000); Kastrinakis G., Phys. Rev. B. 71, 014520 (2005).

[26] Lu D.H. et al., Phys. Rev. Lett. 76, 4845 (1996).

[27] Tamai A. et al., Phys. Rev. Lett. 101, 026407 (2008).

\section{Supplementary Information}

\author{
Appendix A : On the effective interaction \\ $V(q, \omega)$
}

$V(q, \omega)$ of eq. (2) can only be derived in the context of an RPA-type approach, in the spirit of references [15-20] cited in the article. This fact is also emphasized in the recent article by Y. Wang and A.V. Chubukov in Phys. Rev. B 92, 125108 (2015).

\section{Appendix B : On the calculation of the scatter- ing rate}

In the limit $T \rightarrow 0$ the thermal function $X=$ $\operatorname{coth}(\omega / 2 T)+\tanh ((\epsilon-\omega) / 2 T)$ in eq. (4) becomes $X=2$ for $2 T<\omega<\epsilon$, and $X=0$ for $\omega<-2 T$ and $\omega>\epsilon$. Then the integration over $\omega$ - compare with eq. (7) - amounts to

$$
\begin{array}{r}
2 \int_{2 T}^{\epsilon} d \omega \operatorname{Im} V(q, \omega) R(q, \omega) \\
\simeq g R_{0} \ln \left(\frac{K_{q}^{2}+\epsilon^{2}}{K_{q}^{2}+4 T^{2}}\right) \simeq g R_{0} \frac{\epsilon^{2}}{K_{q}^{2}}
\end{array}
$$

for $K_{q}>\epsilon$. The rest of the algebra proceeds as in eq. (8) and onwards. Thus the scattering rate scales like $\epsilon^{2}$ as well, as expected for the FL regime.

We also give some details of the 2-D calculation in the 
main text. The angular integral $I(q, \omega)$ is given by

$$
\begin{gathered}
I(q, \omega)=\int_{0}^{2 \pi} \frac{d \theta s_{o}}{s_{o}^{2}+(c+b \cos \theta)^{2}} \\
=\pi\left(\frac{1}{\sqrt{b^{2}-c^{2}-2 i s_{o} c+s_{o}^{2}}}+\text { c.c. }\right),
\end{gathered}
$$

with $c, b$ as above. Then, taking

$$
\alpha=b^{2}-c^{2}+s_{o}^{2}>0, \quad, \quad \beta=2 s_{o} c, \alpha \gg|\beta|,
$$

we can approximate

$$
I(q, \omega) \simeq 2 \pi\left(\frac{\alpha}{\alpha^{2}+\beta^{2}}\right)^{1 / 2} \simeq \frac{2 \pi}{s_{o}} .
$$

\section{Appendix C : On the calculation of the infinite series for the conductivity}

Setting $Z_{0}=4 N_{F} e^{2} / m$, the n-th term of the conductivity series is given by

$$
\begin{aligned}
& \Delta_{n}=u_{o}^{n} Z_{0} \int_{-\epsilon_{F}}^{\infty} d x \frac{\epsilon_{F}+x}{\left(x^{2}+S^{2}\right)^{n+1}} \\
= & u_{o}^{n} Z_{0}\left(\epsilon_{F} \Gamma_{n}-\epsilon_{F} A_{n}+B_{n}\right),
\end{aligned}
$$

where $x=\epsilon_{k}-\epsilon_{F}$ and

$$
\begin{aligned}
\Gamma_{n}=\int_{-\infty}^{\infty} \frac{d x}{\left(x^{2}+S^{2}\right)^{n+1}} & =\frac{\pi}{2^{n} S^{2 n+1}} \frac{(2 n-1) ! !}{n !}, \\
A_{n} & =\int_{-\infty}^{-\epsilon_{F}} \frac{d x}{\left(x^{2}+S^{2}\right)^{n+1}},
\end{aligned}
$$

$$
B_{n}=\int_{-\epsilon_{F}}^{\infty} \frac{d x x}{\left(x^{2}+S^{2}\right)^{n+1}}
$$

We easily obtain

$$
\Gamma=\sum_{n=0}^{\infty} u_{o}^{n} \Gamma_{n}=\frac{\pi}{\sqrt{S^{2}-u_{o}}},
$$

$$
A=\sum_{n=0}^{\infty} u_{o}^{n} A_{n}=\frac{1}{\sqrt{S^{2}-u_{o}}}\left[\frac{\pi}{2}-\arctan \left(\frac{\epsilon_{F}}{\sqrt{S^{2}-u_{o}}}\right)\right] .
$$

For $n=0$ only, we consider as the upper limit of integration the energy scale $E_{0}>\epsilon_{F}$, instead of infinity (this integral is ultra-violet divergent), and we obtain

$$
B_{0}=\frac{1}{2} \int_{\epsilon_{F}^{2}}^{E_{0}^{2}} \frac{d y}{y+S^{2}}=\frac{1}{2} \ln \left(\frac{E_{0}^{2}+S^{2}}{\epsilon_{F}^{2}+S^{2}}\right) .
$$

For $n \geq 1$ we take infinity as the upper limit of integration, thus obtaining

Hence

$$
\sum_{n=1}^{\infty} u_{o}^{n} B_{n}=\frac{1}{2} \ln \left(\frac{\epsilon_{F}^{2}+S^{2}}{\epsilon_{F}^{2}+S^{2}-u_{o}}\right) .
$$

$$
B=\sum_{n=0}^{\infty} u_{o}^{n} B_{n}=\frac{1}{2} \ln \left(\frac{E_{0}^{2}+S^{2}}{\epsilon_{F}^{2}+S^{2}-u_{o}}\right) .
$$

Putting all these terms together yields the total conductivity given by eq. (18) above. 